\title{
Haptic Rules! Augmenting the gaming experience in traditional games: The case of Foosball
}

\author{
Elia Gatti, Dario Pittera, José Berna Moya, L. and Marianna Obrist
}

\begin{abstract}
Haptic sensations are a crucial aspect of everyday interaction. We touch, lift, move, and probe objects in our everyday activities. However, whilst the importance of haptic feedback has often been emphasized in gaming, haptics has been rarely used to enhance the experience in traditional (non-digital) games. In the last 50 years, technological advancement has allowed an easier access to haptic feedback. Digital games have exploited such access mainly (1) to enhance visual and acoustic feedback, and (2) to reproduce realistic feedbacks in augmented and virtual environments. Here, we re-think haptic feedback by focusing on game augmentation to enrich the gaming experience and gameplay in non-technological games. We describe the design process that led us to define the concept of "haptic rules" as haptic-based enhancement in interference play, where haptic feedback is delivered by users to users within the game as a further mode of interaction. We apply the idea of haptic rules to the game of foosball, evaluating the effect on the gameplay and user experience.
\end{abstract}

\section{INTRODUCTION}

In recent years, haptic interaction has been introduced in a great variety of games (for an inclusive list of examples see [1], [2]). The introduction of haptic interaction, when properly designed, has been reported to increase the likeability of the game and the involvement of the users (e.g., [3], [4]). In fact, industries in the gaming sector are increasingly investing in research for new and engaging forms of haptic interaction (e.g., [5]). However, the gaming and entertainment industry has focused mainly on providing haptic interaction in video games and media (i.e., movies, music), to include more pervasive sensory information in users' experiences (i.e., [69]). In most of these implementations, the delivery of haptic stimuli is dependent on the sensory feedback provided by other sensory modalities. More precisely, to date, haptic feedback has been mainly used (1) to mimic real life interaction where a feeling of contact is not possible (i.e., Virtual Reality) ([10], [11]) or (2) to add strength to existing sensory signals (visual and acoustic feedback), often, but not solely, delivering vibrational patterns to users (for a summary see [12-14]). A good example of the use of haptic technology that falls in both these categories is provided by Israr et al. [15], [16]. The presented technology delivers haptic sensations related to a visual feedback (energy sphere), both mimicking the sensation of touching an inexistent stimulus and delivering vibrations enhancing the characteristics of the stimulus itself.

Rather than enhancing feedback from different sensory modalities, in this paper we describe the design process that led us to conceive a different use of haptic feedback in what is called interference play (gaming experience where interaction between players is shaped in the form of

$\square 11$ authors are with SCHI Lab, School of Informatics, University of Sussex, BN19RH, UK. obrist@ sussex.a.uk. collaboration/opposition ([17], [18])). Particularly, we present a case study where we augment a traditional foosball game by enhancing the game's haptic interaction through the introduction of haptic rules. We implement such rules to give users the possibility to vary the haptic feedback due to the interaction with the foosball during the game, by changing the friction of the opponents' rods when certain targets are hit.

The core idea behind the haptic rules is to provide the users with the means to directly modify the haptic feedback already present in the physical game. In fact, the haptic sensory modality is clearly involved in all the traditional, non-digital games. We receive tactile and kinesthetic sensations when playing cards, interact with a tennis racket, play football and so on. In these examples, haptic sensations are intrinsic in the interaction with physical objects. In our work, we hypothesize that further haptic stimulation, related to the one to the haptic feedbacks arising from the physical interaction with the game, can be incorporated within the rules of non-digital games, providing a new type of gaming experience and enhancing the involvement of users in the gameplay. Here, we selected foosball as our case study. The reasons for choosing foosball were mainly its popularity, simplicity, and the fact that it offers obvious haptic feedback from the interaction with the rods. Such interaction can be easily modified without the use of complicated technology.

In sum, this paper represents an original attempt to: (1) redefine traditional game experiences through the introduction of new haptic rules and (2) identify design opportunities for stimulating richer gaming experiences through sensory enhancement, which in our use case is haptic feedbacks, but does not need to be limited to that modality.

\section{GAME AUGMENTATION}

The joy and excitement of interacting with the physical world, typical of traditional, physical games, cannot be easily replicated or simulated in a computerized gaming environment [19], [20]. For this reason, research in human-computer interaction is increasingly integrating physicality in game design rather than discarding it in favor of a purely digital gaming environment. The augmentation of physical games is often achieved through visual and acoustic sensory enhancements (e.g., [20], [21]), and virtual and augmented reality [22].

"Augmented games", as well as "pervasive games" and "exertion games" are all examples of such enhancements. Ishii et al. [20], for instance, investigated the augmentation of a ping pong table by adding digital layers of graphics and sound. In their game augmentation, the authors created seven different 
game modalities, each with a different set of rules and goals for the users to achieve. Also, Altimira and colleagues [23] used visual augmentation to modify the game of table tennis. In this case, the authors modified the field size during the game [23]. Jebada et al. [22] focused instead on enhancing billiard playing through augmented reality and wearable computing. More recently, Kajastila and colleagues [21] used a projector to deliver visual stimuli to users climbing and bouldering in an indoor gym. In this case, different games were created around such feedback, enhancing the experience and the performance of the climbers. Pervasive games are another example of games that offer additional engaging sensory feedback to users, particularly among augmented reality (e.g., [24], [25]) and augmented tabletop games [26]. Finally, what have been called "exertion games" [27] also strongly relate to the augmentation of physical games. In exertion games, the gameplay is enhanced by paying attention to different aspects of the interaction between users, such as their social dynamics, the pain, and their exertion trajectories [28].

Although the above-mentioned works are related to the concept of haptic rules proposed in this paper, it is worth considering the differences between our approach and the aforementioned examples. Introducing haptic rules aims to augment a physical game through technology. However such augmentation 1) does not change the purpose of the game (as instead happens in ([20], [21]) nor 2) adds any information or tips about the gameplay (as in the case of [22]). Furthermore, in our implementation 3) we do not add a new layer of sensory stimulation (as in, e.g., [12]), but rather modify an existing stimulus arising from the physical interaction with the game.

\section{ENHANCING THE USER'S EXPERIENCE}

The ultimate aim of augmenting a game is to enhance the user's experience in the gameplay. Here we describe the process we followed in designing haptic rules based on the foosball case study. Figure 1 shows the six main steps in the process, adapted from [29].

Figure 1: Design process to establish the haptic rules (adapted from [29]).

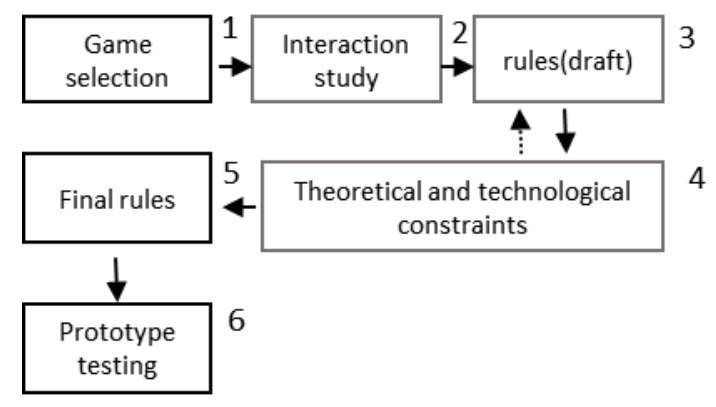

In the first step, "game selection", the game in which to implement the rules is selected. Subsequently, the interaction between users and the selected game is investigated, in order to identify the relevant haptic interactions. When the most relevant haptic interaction is clear, a first set of rules is proposed. Rules are expected to have an effect on user's haptic interaction. In the next step, the set of rules is confronted with design guidelines and benchmarks on game design from literature (e.g., [30]). Rules feasibility is also considered from an engineering standpoint. If the rules contradict good design practice or are not feasible, a new set of rules is proposed (iteration to step 3). The process continues until a polished and applicable set of rules is available. In a final step, a prototype of the augmented game is built and tested in order to validate the effect of the augmented game on the gaming experience and gameplay.

\section{THE HAPTICALLY AUGMENTED FOOSBALL}

We investigated the interaction between users and the game by interviewing 10 frequent players (playing at least once a week, mean age $30.24 \pm 2.14$ ) on their gaming experience. Unsurprisingly, the most relevant haptic interaction resulted to be the manipulation of the table rods by the users. A set of rules was therefore proposed during a brainstorming session. Particularly, 2 rules were proposed, both impacting on the user's interaction with the rods. The rules were subsequently assessed in terms of feasibility (engineering constraints) and expected effect on the gameplay. On the latter, particular attention was posed to guidelines expressed in [30] and [31] for effective game design (e.g., Players should receive simple and efficient access to information. Feedback should be immediate and continuous). One rule (the rod's friction augment depending on the position of the ball on the field") was discarded as it required accurate tracking of the ball on the field. The remaining rule ("rod's friction can be altered hitting targets on the side of the goal") was iteratively modified and divided in three different rules. Sections A and B describe, respectively, the final haptic rules and the implementation of the augmented foosball.

\section{A. The Game}

The two main rules of traditional foosball (at a nonprofessional level) are: (1) score more goals than the opponent (usually for a maximum of six or ten goals) (2) without spinning the rods (more than 360 deg.). Those rules were kept consistent in our augmented version of the foosball game and extended with new haptic rules. We have chosen foosball as a target game to investigate sensory enhanced gaming experiences because it naturally enhances the already physically engaging gameplay. We designed a modified foosball table Figure 2, Figure 3) that enabled the enhancement of the game with new haptic rules. The haptic rules were defined as follows:

- When the plate on the right side of the opponent's goal is hit, the friction on the opponent's rod will increase, therefore constraining the opponents' movements, making harder for them to move the players.

- When the plate on the left side of the opponent's goal is hit, any friction previously applied on the own team's rod will be released.

- After each goal, any friction active at that moment will be released for both teams.

\section{B. Game adjustment and implementation}

We used four piezoelectric sensors (RVFM ABT-441-RC) at the back of each of the 2 metal plates $(8 \mathrm{~cm} \mathrm{X} 22 \mathrm{~cm})$ on the right and left side of the playing field. The plates were coloured either green or red. The sensors were used to detect hits on the plates. Impact with sufficient force $(0.33 \mathrm{~N})$, the red metal plate on the right side of the opponents' goal activated 
mechanical clamps installed on the opponents' rods, which exerted friction that in turn restricted the users' movements. Conversely, hitting the green metal plate on the left side allowed the users to release the friction and their own rods and regain full control. Hitting the red plate also triggered on a LED light located in the goal hole to provide visual feedback on the activated block.

Figure 2: The goal of the haptically augmented foosball. On the side of the goal, it is possible to see the sensorized plates. Hitting the red plate blocks the opponents' rods, hitting the green plate releases the friction previously applied on one's own team.

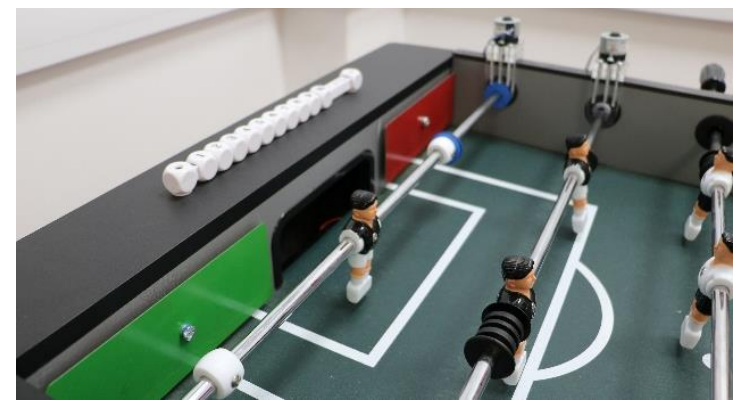

The U-shaped clamps were manufactured using $12 \mathrm{~mm}$ clear acrylic. A linear actuator was attached (Crouzet Mfr. Part No.80 910 002) to the clamps in order to produce a vertical displacement $(4 \mathrm{~mm}$ ) by pivoting on the edge of the foosball table. PVC rubber in the inner rod hole provided friction against the rod. Once the clamps were activated, the users had to apply $\mathrm{X} \mathrm{N} / \mathrm{mm}$ linear force to move the rods or wait for release. Sensor readings and stepper activation were controlled via open loop control (running on an Arduino Leonardo Board with 4xL293D H-bridges).

Figure 3: One of the acrylic clamps used to increase the friction on the users' rods. The clamp has a linear actuator installed on the top connected with a screw. Hitting the red plate sends signals to the actuator, which causes the screw to pull the clamp upwards, making harder to move the rods.

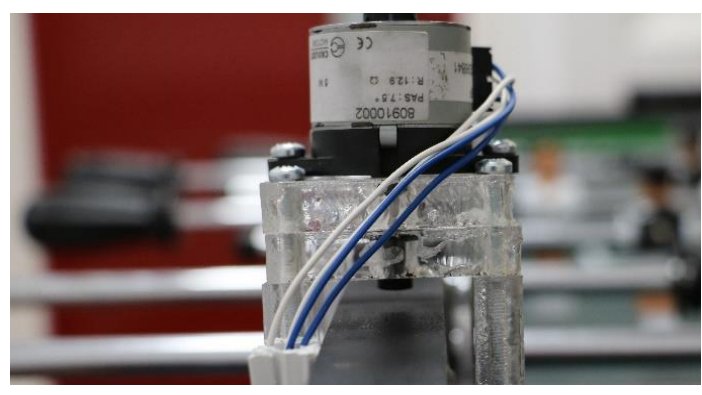

\section{USER STUDY}

To assess the effect of the haptic rules on the users' experience and engagement in the gameplay, we designed a within-subject experiment where two conditions: traditional and augmented foosball game, were compared. Users played in a team against two experimenters. The experimenters tried to adjust their gameplay to the users' prowess in the game by never exceeding 2 goals of difference from the opponents.

Please note that the experimenters were effectively "actors" in the game to ensure all users to be exposed to the same conditions and minimize variability. We wanted the ratings of the users not to be biased by their interaction with their opponent, which would have been the case when the opponent was too good or too bad. By keeping the opponent constant, and able to adapt the game depending on the user, we believe we were able to rule out such component from our results.

\section{A. Study setup and procedure}

In our user study, users were invited to play two matches. In condition 1, users played following the traditional foosball game rules, with not modified haptic feedback. In condition 2 , users played the game with the additional haptic rules, the actuators and the sensor plates turned on. The order of the conditions (with or without haptic rules) was randomized and counterbalanced across pairs of users. Each match terminated after 10 minutes (a timer was set at the beginning of each match) or earlier if one team reached 10 goals (maximum per match).

The foosball table was set up in a dedicated room that allowed for enough movement around the table as well as a comfortable atmosphere for playing the game. After signing the informed consent, users were instructed about the rules of the augmented foosball. Additionally, the rules were printed on a sign next to the foosball table and explained at the beginning of the session. Each game started with a three minutes' warm-up session to familiarize the users with the foosball table (traditional and augmented). The session also allowed the experimenters to adapt their gameplay to the users' skills level. At the start of the first match, users were asked to choose their preferred position on the table (attack or defend user in their team) and keep this position for the rest of the experiment in both conditions.

\section{B. Users}

In total, we recruited 16 users $(28.4 \pm 4.14$ mean age, 12 males). The experiment lasted on average 45 minutes. Users were recruited from the local University through printed and electronic advertisements on notice boards. We recruited users in pairs. The same pair experimenters (both males) played against each team of users.

\section{Gameplay logging}

Behavioral data were recorded during the experiment. In particular, all the main events of the gameplay were logged for each team (white and black team):

- Scoring a goal

- Blocking the opponent

- Releasing the block from the opponent

The duration of each match and the gap between each event were also recorded. This allowed us to extract important information concerning the gameplay and the behavior of the users during the game. Moreover, each game was video recorded in order to achieve a better understanding of the logged events.

\section{Questionnaire on the foosball matches}

After each match, users completed a questionnaire that included the outcome of the game (if they won or lost) and six statements on the experience of the game. users were asked to rate how much they agreed or disagreed with the following statements on a scale from 0 (not agree at all) to 7 (completely 
agree): Q1: I enjoyed playing; Q2: I felt connected with my teammate; Q3: I felt connected with my opponents; Q4: It was a stimulating experience; Q5: It was an interesting experience; Q6: It was a frustrating experience.

\section{RESULTS}

We analyzed all the data for each team (i.e., gameplay) and individually for each user (i.e., ratings). The data analysis of the questionnaire ratings and gameplay analysis and graphics were performed using the statistical software R.

\section{A. Gameplay logging}

By recording the main events during each match, we were able to assess differences in the gameplay between the haptic rules and the control condition. In general, matches played following the haptic rules lasted longer $(8.7 \pm 1.2$ minutes $)$ than matches played with the traditional foosball $(7.23 \pm 1.61$ minutes). We ran a non-parametric version of paired t-test (Wilcoxon test) to assess whether the difference was statistically significant, with negative results $(\mathrm{W}=14, \mathrm{p}=$ 0.06). No differences were found in the number of goals scored by each team $(\mathrm{W}=44.5, \mathrm{p}=0.19)$. In our analysis, we also compared the number of times each plate was hit in each condition by each team. Chi-squared test was computed on the number of time each plate was hit in each condition.

Results showed that the number of time a plate was hit strongly depended on the condition (chi-squared $=13.44$, df $=3$, p-value < 0.01) $(4,1)$. However, it is worth to consider that the gameplay from the pair of experimenters was likely to be biased by their knowledge of the purpose of the study, as well as by the attempt of self-imposing the restriction to not exceed the 2 goals difference. We therefore repeated the analysis on the number of hits by excluding the plates hit by the experimenters' team. In this case, results showed no significant difference between the two conditions (chisquared $=0.34, \mathrm{df}=1, \mathrm{p}$-value $=0.55)$.

TABLE I

\begin{tabular}{|l|l|l|l|l|}
\hline \multirow{2}{*}{} & \multicolumn{4}{|c|}{ Hits } \\
\cline { 2 - 5 } & Up-left & $\begin{array}{c}\text { Bottom- } \\
\text { Right }\end{array}$ & $\begin{array}{c}\text { Top- } \\
\text { Right }\end{array}$ & Bottom-Left \\
\hline Control & 29 & 54 & 47 & 41 \\
\hline Haptic & 60 & 41 & 38 & 39 \\
\hline
\end{tabular}

Table 1: Overview of the number of hits on each corner plate for control (traditional foosball) and haptic (augmented foosball) condition.

Figure 4. Representation of the number of hits for each plate of the foosball for control (traditional foosball in light blue) and haptic rules conditions. The radius of each circle is proportional to the number of hits.

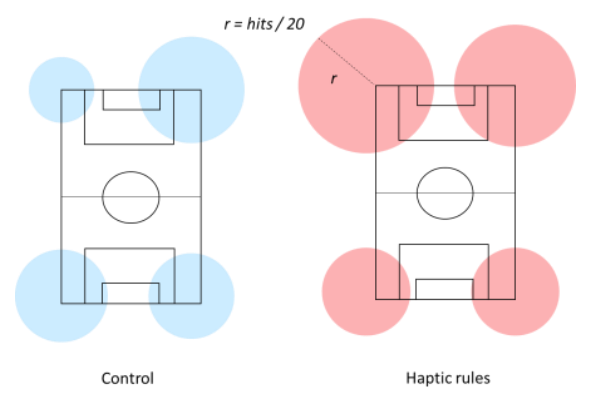

\section{B. Questionnaire on the foosball matches}

To directly assess the experience of the users, we analyzed their responses to the questionnaire described earlier. Table 2 summarizes the mean and standard deviation for each item of the questionnaire in both conditions. To assess whether those differences were statistically significant we run a Wilcoxon test for each item of the questionnaire. The analysis shows a statistically significant difference between conditions for 4 questions out of 6 (Q2 to Q6, p $<0.05$ in all cases). Q1 (• I enjoyed playing', $\mathrm{p}=0.47$ ), and Q6 ('It was a frustrating experience', $\mathrm{p}=0.31$ ) did not show statistically significant differences between conditions 5 .

Finally, we examined the correlation between the expertise reported by the users and the ratings for each question, under the hypothesis that skilled users could go through a different experience than beginners. The level of expertise was based on preliminary questions asked before matches, i.e.: how often they played and how good they and their friends considered them to be (from 1 to 7 on a Likert scale). Results for these correlations were however negative, showing the highest correlation coefficient as -0.2 , not significant $(\mathrm{p}=$ 0.4). Furthermore, ratings from users playing in the attack position did not differ from those of users playing in defense in the control condition. However, in the augmented condition, defenders generally found the experience significantly more stimulating than attackers (Wilcoxon test, $\mathrm{p}<0.05)$.

TABLE II

\begin{tabular}{|l|l|l|}
\hline \multicolumn{3}{|c|}{ Questionnaires results } \\
\hline Question & Control & Haptic \\
\hline Q1 & $5.6 \pm 0.8$ & $5.4 \pm 0.7$ \\
\hline Q2 & $5.1 \pm 1$ & $6.1 \pm 0.6$ \\
\hline Q3 & $4.6 \pm 1.3$ & $5.3 \pm 0.9$ \\
\hline Q4 & $5 \pm 1$ & $6.3 \pm 0.7$ \\
\hline Q5 & $5.2 \pm 0.7$ & $6.5 \pm 0.6$ \\
\hline Q6 & $2.6 \pm 1.2$ & $3.4 \pm 1.8$ \\
\hline
\end{tabular}

Table 2: Mean and standard deviation for the 6 items of the questionnaire evaluating the experience of users after each condition (haptic control) and for each condition after a week time (haptic-post, control post).

\section{DISCUSSION}

In the present work, we augmented the game of foosball by introducing haptic rules. We defined haptic rules as a new layer of rules that regulate the haptic feedback arising from the physical interaction with the game. The process leading to the definitions of the haptic rules is shown in Figure 2. In this section, we discuss our finding on the effect of the haptic rules on users' experience and gameplay.

\section{A. Feeling connected}

Users significantly reported feeling more connected with their teammate and their opponents (Q2 and Q3). This effect is likely to be due to mechanisms such as shared reward, shared punishment, and empathy [32]. When users were blocked alongside their teammates, they both shared the same negative 
experience (punishment). When they were released, they shared the same positive experience (reward). We suppose that sharing rewards and punishments together with teammates led to stronger bonding. In the same way, seeing members of another group experiencing an unfavorable situation could have led to empathy.

Figure 5. Pooled in ratings from each user $(\mathrm{N}=16)$ for each question in the control (traditional foosball) and haptic (augmented foosball) conditions.

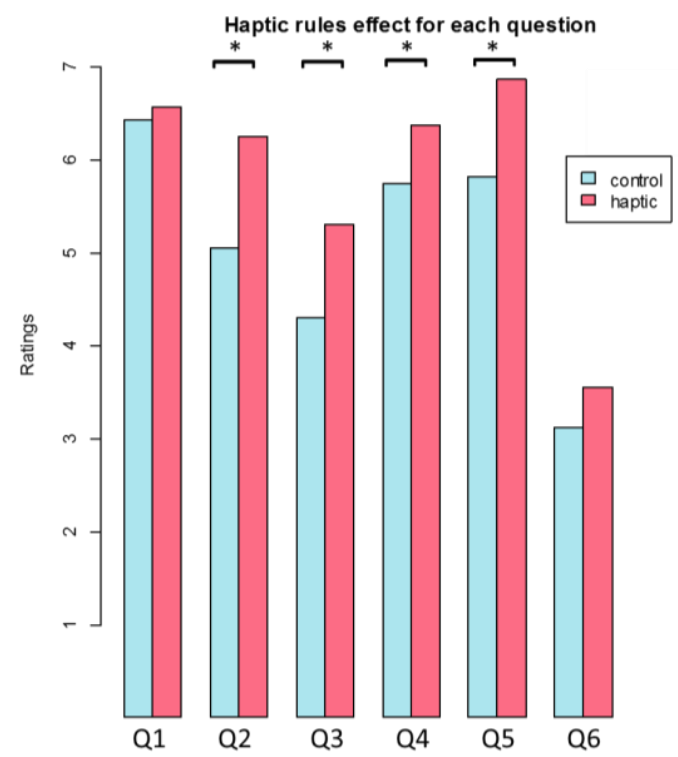

The questionnaire feedbacks shows that the augmented foosball was a more interesting and stimulating experience when compared with the traditional version of the game. Although these results seem to support the idea of an overall improvement of the gaming experience through the introduction of the haptic rules, it is worth noting that such higher values might be due to the novelty and surprise represented by the augmented foosball, as surprise has been shown to be a powerful factor in user experience [33].

Finally, Q1 and Q6 (enjoyment and frustration) did not differ between control and haptic conditions (that are: traditional and augmented foosball). This could be due to an effect of order in the presentation of the two conditions. Since the order of the condition was balanced across the 16 users, 4 of the eight matches happened to have the control condition first. The traditional version of the foosball game itself is both engaging and enjoyable. Thus, we suppose that users rated it very high on the Likert scale when experiencing it first, therefore producing a ceiling effect [34]. Indeed, a Wilcoxon test showed that users experiencing the control condition first, rated such condition significantly higher than users starting the experiment with the augmented foosball $(\mathrm{p}<0.05)$. Moreover, Wilcoxon test showed significant difference in Q1 ratings for those cases in which the haptic version of the game was presented first $(\mathrm{p}<0.05)$. Enhancement in enjoyment during the game could be due to different causes. The surprise and the excitement of a new game could have played a role in the users' ratings. However, it is likely that the sense of control generated by blocking the opponent could have been a driving factor in the users' experience. In fact, the users taking part in our study were not particularly skilled users.
Being able to gain control over a game in which otherwise they would have little prowess could have greatly improved the enjoyment of the game. Indeed, previous studies showed the importance that "feeling in control" of the game has in creating an enjoyable experience [35]. Altimira et al. [23] also showed that balancing the game based on the skill level of the user has a positive effect on the user's engagement and experience. Finally, it is worth noticing that participants did not find the haptic version to be more frustrating than the control one. In this case, no effect of the presentation was shown (Wilcoxon test, $\mathrm{p}=0.23$ ), supporting the idea that the haptic rules improved the game experience, or at least, did not spoil it.

\section{B. Effects on the gameplay}

Interestingly, no statistically significant differences on the recorded gameplay events were found between the augmented and the control conditions. Such result is somewhat surprising, considering the differences in the reported experience, as well as the significant change in the physical interaction with the game that was implicit in the augmented condition. Whereas the lack of statistical evidence might simply indicate that users did not approach the game differently in terms of strategy and game behavior, reports from users after the experiment pointed to the opposite direction. In fact, after the experiment, many users reported they aimed purposely for the block plates. We therefore think that the main reason behind the lack of statistical evidence can be related to the relatively low skills of the users taking part to the game. Future evaluations of the augmented foosball will need to be open to a higher number and a wider variety of users. To support the claim that participants aimed for the plate in the haptic condition (augmented foosball), and only hit the plate by mistake in the control one, we computed the probability of scoring after hitting the left side of the goal in both conditions, excluding the experimenters from the analysis. As expected, the probability of a goal after hitting the block plate raised considerably in the augmented condition (probability haptic 0.126 , probability control 0.096 , frequency haptic 24 , frequency control 10). This evidence, if combined with the users' feedback, allows us to support the idea of an effect of the haptic rules on the gameplay. However, there is an element of serendipity in hitting the plate, which requires further investigation in order to reach stronger evidence for this particular strategy in the gameplay.

\section{CONCLUSION}

In the present paper, we introduce what we called haptic rules, simple technological augmentations enabling users to control the experienced haptic feedback arising from the physical interaction with the game. Results from our case study showed that the haptic rules enhanced the users' experience with the game, also making people feel more connected with teammates and opponents.

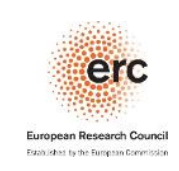

\section{ACKNOWLEDGMENTS}

This project has received funding from the European Research council (ERC) under the 
European Union's Horizon 2020 research and innovation programme under grant agreement No 638605.

\section{REFERENCES}

[1] D. Morris.. "Haptic Battle Pong: High-Degree-of-Freedom Haptics in a Multiplayer Gaming Environment. Experimental Gameplay Workshop", GDC. In Proc. Experimental Gameplay Workshop, 2004, GDC.

[2] W. Piekarski, B. Thomas. ARQuake: "The Outdoor Augmented Reality Gaming System”. Commun. ACM 45, 2002, 36-38.

[3] A. Hamam, M. Eid, A. El Saddik, and N. D. Georganas. "A Quality of Experience Model for Haptic User Interfaces". In Proceedings of the 2008 Ambi-Sys Workshop on Haptic User Interfaces in Ambient Media Systems (HAS '08). ICST (Institute for Computer Sciences, Social-Informatics and Telecommunications Engineering), ICST, Brussels, Belgium, Belgium, Article1, 2008, 1-6.

[4] R. Bernhaupt. User Experience Evaluation in Entertainment and Games. Springer Berlin Heidelberg, Berlin, Heidelberg, 2011,716-717.

[5] Neurodigital Technologies. 2012. Glove One. Website. (15 July 2012). https:https://www.gloveonevr.com/.

[6] O. E. A. Taylor, S. C. Nanayakkara, L. L. Wyse, S. H. Ong, K. P. Yeo, and G. H. Tan. "Haptic chair sound enhancing system with audiovisual display". US Patent 8,638,966, 2014

[7] F. Arafsha, K. M. Alam, and A. E. Saddik. "EmoJacket: Consumer centric wearable affective jacket to enhance emotional immersion." In Innovations inInformation Technology (IIT), 2012 International Conference on. 350-355.

[8] C. Thorner. "Universal tactile feedback system for computer video games and simulations". (Nov. 13 2012). US Patent 8,308,558, 2012

[9] Mohamad Eid, Ahmad El Issawi, and Abdulmotaleb El Saddik. 2014. Slingshot 3D: A synchronous haptic-audio-video game. Multimedia Tools and Applications 71, 3 (2014), 1635-1649.

[10] E. Kruijff, A. Marquardt, C. Trepkowski, J. Schild, and A. Hinkenjann. "Enhancing User Engagement in Immersive Games through Multisensory Cues". In Games and Virtual Worlds for Serious Applications (VSGames), 7th International Conference on. 2015, 1-8.

[11] Y. Kusunose, Y. Ishibashi, N. Fukushima, and S.Sugawara. "QoE Assessment in Networked Air Hockey Game with Haptic Media". In Proceedings of the 9th Annual Workshop on Network and Systems Support for Games (NetGames '10). IEEE Press, Piscataway, NJ, USA, Article $11,2010,1-2$ pages.

[12] M. A. Srinivasan and C. Basdogan. 1997. "Haptics in virtual environments: Taxonomy, research status, and challenges". Computers and Graphics 21, 4, 1997, 393 - 404.

[13] A. E. Saddik. "The Potential of Haptics Technologies". IEEE Instrumentation Measurement Magazine 10, 1, 2007, 10-17.

[14] D. Chang. 2002. "Haptics: gaming's new sensation". Computer 35, 2002, 84-86.

[15] A. Israr and I. Poupyrev. 2011. "Tactile Brush: Drawing on Skin with a Tactile Grid Display". In Proceedings of the SIGCHI Conference on Human Factors in Computing Systems (CHI '11). ACM, New York, NY, USA, 2019-2028.

[16] A. Israr, S. Zhao, K. McIntosh, J. Kang, Z. Schwemler, E. Brockmeyer, M. Baskinger, and M. Mahler. "Po2: Augmented Haptics for Interactive Gameplay". In ACM SIGGRAPH 2015 Emerging Technologies (SIGGRAPH '15). ACM, New York, NY, USA, Article 21, 2015 1-10.

[17] J.L. Frost, S.C. Wortham, R.S. Reifel Play and Child Development, Prentice Hall, Pearson/Merrill, 2008.

[18] F. Mueller, M. Gibbs, F. Vetere, S. Agamanolis, D. Edge. "Designing mediated combat play". In: Proceedings of the 8th International Conference on Tangible, Embedded and Embodied Interaction-TEI'14. ACM Press, New York, New York, USA, 2014, 149-156.
[19] T. Iwata, T. Yamabe, M. Polojärvi, and T. Nakajima. "Traditional Games Meet ICT: A Case Study on Go Game Augmentation". In Proceedings of the Fourth International Conference on Tangible, Embedded, and Embodied Interaction (TEI '10). ACM, New York, NY, USA, 2010, 237-240.

[20] H. Ishii, C. Wisneski, J. Orbanes, B. Chun, and J. Paradiso. "PingPongPlus: Design of an Athletic-tangible Interface for Computer-supported Cooperative Play". In Proceedings of the SIGCHI Conference on Human Factors in Computing Systems (CHI '99). ACM, New York, NY, USA, 1999, 394-401.

[21] Raine Kajastila, Leo Holsti, and Perttu Hämäläinen. "The Augmented Climbing Wall: High-Exertion Proximity Interaction on a Wall-Sized Interactive Surface". In Proceedings of the 2016 CHI Conference on Human Factors in Computing Systems (CHI '16). ACM, New York, NY, USA, 2016, 758-769.

[22] T. Jebara, C. Eyster, J. Weaver, T. Starner, and A. Pentland.. "Stochasticks: augmenting the billiards experience with probabilistic vision and wearable computers". In Wearable Computers, 1997. Digest of Papers. First International Symposium on. 1997, 138-145.

[23] D. Altimira, F. Mueller, J.Clarke, G. Lee, M. Billinghurst, and C. Bartneck.. "Digitally Augmenting Sports: An Opportunity for Exploring and Understanding Novel Balancing Techniques". In Proceedings of the 2016 CHI Conference on Human Factors in Computing Systems (CHI '16). ACM, New York, NY, USA, 2016, 1681-1691.

[24] T. C. T. Qui, T. H. D. Nguyen, A. Mallawaarachchi, K. Xu, W. Liu, S. P. Lee, Z. Y. Zhou, S. L. Teo, H. S. Teo, L. N. Thang, Y. Li, A. David Cheok, and H. Kato. "Magic Land: Live 3D Human Capture Mixed Reality Interactive System". In CHI '05 Extended Abstracts on Human Factors in Computing Systems (CHI EA '05). ACM, New York, NY, USA, 2005, 1142-1143.

[25] M. Hansen, J. H. Petersen, C. L. Olander, N. Broks $\varnothing$, M. Olsen, J. G. Dinesen, and K. B. Schiøler. Augmented Reality Games. Doctoral. Dissertation. 2011.

[26] Carsten Magerkurth, Richard Stenzel, and Thorsten Prante. 2003. "STARS - A Ubiquitous Computing Platform for Computer Augmented Tabletop Games". In Adjunct Proceedings of the Fifth International Conference on Ubiquitous Computing (UBICOMP03) Springer, 2003, 267-268.

[27] J. Marshall, F.Mueller, S. Benford, S. Pijnappel, "Expanding exertion gaming", International Journal of Human-Computer Studies, Volume 90, 2016, 1-13.

[28] F. Mueller, M. R.. Gibbs, and F. Vetere. Taxonomy of exertion games. In Proceedings of the 20th Australasian Conference on Computer-Human Interaction: Designing for Habitus and Habitat. 2008, 263-266.

[29] E. Gatti. Feel and sense the product: experimental based optimization methodology. Doctoral dissertation, Italy. 2014.

[30] A. E. Saddik. "The Potential of Haptics Technologies". IEEE Instrumentation Measurement Magazine 10, 1, 2007, 10-17.

[31] S. Hinske, , M. Langheinrich, M. Lampe: "Towards Guidelines for Designing Augmented Toy Environments." In: v.d. Schijff, J., Marsden, G., Kotze, P. (eds.): Proc. of the 6th ACM Conf. on Designing Interactive Systems (DIS 2008), ACM Press, New York, NY, USA 2008

[32] V. Gallese. "The roots of empathy: the shared manifold hypothesis and the neural basis of intersubjectivity". Psychopathology 36, 4, 2003 , 171-180.

[33] M. Hassenzahl and N. Tractinsky. User experience-a research agenda. Behaviour information technology 25, 2, 2006, 91-97.

[34] D. L Clason and T J Dormody. “Analyzing data measured by individual Likert-type items". Journal of Agricultural Education 35, 1994,1- 4.

[35] Hannu Korhonen, Markus Montola, and Juha Arrasvuori. 2009. Understanding playful user experience through digital games. In International Conference on Designing Pleasurable Products and Interfaces, Vol. 2009. Citeseer. 